\title{
Inherited and flatband-induced ordering in twisted graphene bilayers
}

\author{
Lennart Klebl ${ }^{1}$ and Carsten Honerkamp ${ }^{1}$ \\ ${ }^{1}$ Institut für Theoretische Festkörperphysik, RWTH Aachen University, \\ and JARA Fundamentals of Future Information Technology, Germany
}

(Dated: July 24, 2019)

\begin{abstract}
The nature of the insulating and superconducting states in twisted bilayer graphene systems is intensely debated. While many works seek for explanations in the few flat bands near the Fermi level, theory and a number of experiments suggest that nontwisted bilayer graphene systems do exhibit - or are at least close to - an ordered, insulating ground state related to antiferromagnetic ordering. Here we investigate in which ways this magnetic ordering scenario is affected by the slight twisting between the layers. We find that at charge neutrality the ordering tendencies of twisted systems interpolate between those of untwisted AA and AB stacked bilayers at intermediate temperatures, while at lower temperatures of the order of typical flat-band dispersion energies, the ordering tendencies are even enhanced for the twisted systems. The preferred order at charge neutrality still exhibits an antiferromagnetic spin arrangement, with ordered moments alternating on the carbon-carbon bonds, with an enveloping variation on the moiré scale. This ordering can be understood as inherited from the untwisted systems. However, even in the RPA analysis, the possible low-energy behaviors are quite versatile, and slight doping of one or more electrons per moiré cell can take the system into a, potentially flat-band induced, ferromagnetic phase.
\end{abstract}

The discovery of superconductivity in the vicinity of partially insulating correlated-metal states in twisted bilayer graphene samples has triggered numerous experimental1 14 and theoretical efforts. According to numerous theoretical works ${ }^{5-13}$, in parts already before the discovery of superconductivity in these systems, the slight twisting leads to a remarkable modification of the lowenergy spectrum, with 4 rather flat bands which are separated from the other bands quite well in the case of magic twist angles. Many explanation attempts 14 24 involve enhanced electron correlation effects due to this condensed low-energy spectrum.

Ab-initio estimates for the interactions in and between the Wannier states of the flat bands come up with quite large values exceeding the bandwidth of the flat bands 1112 . However it can be expected these large values will be screened down by the remnant $\pi$-band spectrum ${ }^{25}$. In general, it is believed that focusing on the flat bands is legitimate as they are separated from the rest of the $\pi$-band spectrum by a small energy gap of a few meVs, but whether this is sufficient to exclude decisive influence by the large rest of the spectrum, is hard to know without well-defined calculations.

In this paper, we avoid this uncertainty by working with the full $\pi$-bands. On this larger energy scale, the interactions are smaller or comparable to the band width. In fact, the strength of onsite and nonlocal interactions for $\pi$-electrons has been a resurfacing topic over quite some time in solid state theory 26-29. More recently, the interaction as a function of distance has been analyzed starting from ab-initio, also taking into account environmental screening 30 . So, as a starting point, the $\pi$-band model is quite well-defined and should allow one to obtain almost quantitative insights, with the main uncertainties arising from the theoretical treatment of the nonlocal moderate interactions within this model. A second reason for considering the full $\pi$-bandwidth is that a few years ago, nontwisted, freely suspended bi- and trilayer graphene systems were investigated intensely, as experiments showed the opening of a likely interaction-induced gap of a few meV width at low temperatures 31 . The theoretical understanding is that this gap was likely due to the onset of antiferromagnetic order in the layers 35 with staggered moments within one layer and unequal absolute magnitude on the two sublattices. Note that in effective models for AB-stacked bilayer where only the two bands touching the Fermi level are taken into account, this state comes in disguise as intralayer ferromagnetic inter-layer antiferromagnetic 'layer antiferromagnet' (LAF) ${ }^{39}$ 41, as only one sublattice per layer is considered. More recently, experiments on Bernalstacked multilayers ${ }^{42}$ 44 exhibited quite the same gap phenomena with clear increase of the gapping temperature up to $100 \mathrm{~K}$ and, not unexpectedly based on the band structure, an even-odd effect in the low- $T$ conductance in the number of layers 43 . It should however be mentioned that not all experiments on bilayers 45,47 showed a full gap at low $T$ and also theoretically, there are other options $\$ 0|48| 49$. So it may well be some these layered graphene systems are only on the verge of such ordering instability and additional details of the samples or the environment decide about its actual occurrence and type of order.

Now, given the observation of insulating states in twisted bi- and multilayer systems one may ask if these states are in any way related to the potential ordering instability in the nontwisted systems. Hence, the idea of the current manuscript is to use an approach that produces the AF instability as a likely candidate for the nontwisted bilayer systems and to see what the changes are if a slight twisting is introduced. In fact, the occurrence of an antiferromagnetic ordering on the $\mathrm{C}-\mathrm{C}$ bond scale for the twisted bilayer is already shown in self-consistent studies for onsite interactions in Ref. [50. Here we ask 
if this instability is stronger in the twisted system compared to the $\mathrm{AB}$ or $\mathrm{AA}$ bilayer and analyze the scenario in more depth, e.g. regarding the consequences of doping.

In order to make this comparison, we set up the nontwisted and twisted unit cells of equal size and derive the non-interacting band structure of the moire superlattice. Then we compute the relevant particle-hole diagrams at zero momentum transfer, from which we can infer instabilities toward magnetic order using the random phase approximation (RPA), which in this case can be understood as a generalized Stoner theory. We compare the strength of the magnetic ordering as a function of the temperature and band filling and relate it to nontwisted bilayer systems. Then we also study the ordering pattern within the moire unit cell and use this as estimate for the ordered moment below the ordering transition. Inserting this as meanfield into the electronic dispersion yields renormalized, split-up bands, which can be related to the experimental observation of insulating states.

\section{MODEL}

We start with an AA bilayer of carbon sites spanned by the two in-plane Bravais lattice vectors $\boldsymbol{l}_{1}=$ $(\sqrt{3} / 2,3 / 2,0)$ and $\boldsymbol{l}_{2}=(\sqrt{3}, 0,0)$ and two sites per unit cell separated by the vector $(0,1,0)$, all measured in units of the C-C bond length $a_{0}=0.142 \mathrm{~nm}$. The 'vertical' distance between the two layers is given by $\sqrt{51}$ the shift vector $0,0, d)$ with $d=0.335 \mathrm{~nm}=2.36 a_{0}$. The large unit cell of the twisted bilayer system is obtained by defining two superlattice vectors $\boldsymbol{L}_{1}=n \boldsymbol{l}_{1}+m_{2}$ and $\boldsymbol{L}_{2}$ rotated by 60 degrees with respect to $\boldsymbol{L}_{1}$. Then one of the two layers is rotated by the twist angle $\theta=\arccos \frac{m^{2}+n^{2}+4 m n}{2\left(m^{2}+n^{2}+m n\right)}$ around an AA lattice carbon site. For the magic angle $\theta=1.05^{\circ}$ we use $n=31$ and $m=32$ (denoted 31/32-system). This produces a moiré unit cell of 11908 carbon sites. Next we set up the Koster-Slater tightbanding Hamiltonian matrix as described in Refs. 5 and 51, including the corrugation detailed in the appendix of Ref. 11. From that we obtain for each $\boldsymbol{k}$ in the folded Brillouin zone the band energies $\epsilon_{b}(\boldsymbol{k})$ and eigenvectors $u_{i b}(\boldsymbol{k})$ for band $b$ and site index $i$ inside the moiré unit cell. In Fig. 1 we show the low-energy band structure in the moiré Brillouin zone for the $31 / 32$-system as well as the non-twisted $\mathrm{AA}$ and $\mathrm{AB}$ bilayers using the same moiré supercell. One can clearly see the formation of the flat bands in the twisted system in contrast with linear or quadratic wider bands in the case of $\mathrm{AA}$ and $\mathrm{AB}$ bilayers (both with constant interlayer distance). Due to the backfolding, the AA bilayer shows a numerous linear Fermi level crossings in the small moiré Brillouin zone, while the $\mathrm{AB}$ bilayer exhibits quadratic band crossing points at $K$ and $K^{\prime}$. These are actually split up into separate Dirac points on a low energy scale not visible in this plot, due to the remote hoppings included in this calculation.

Next, we consider Hubbard onsite interactions for elec-
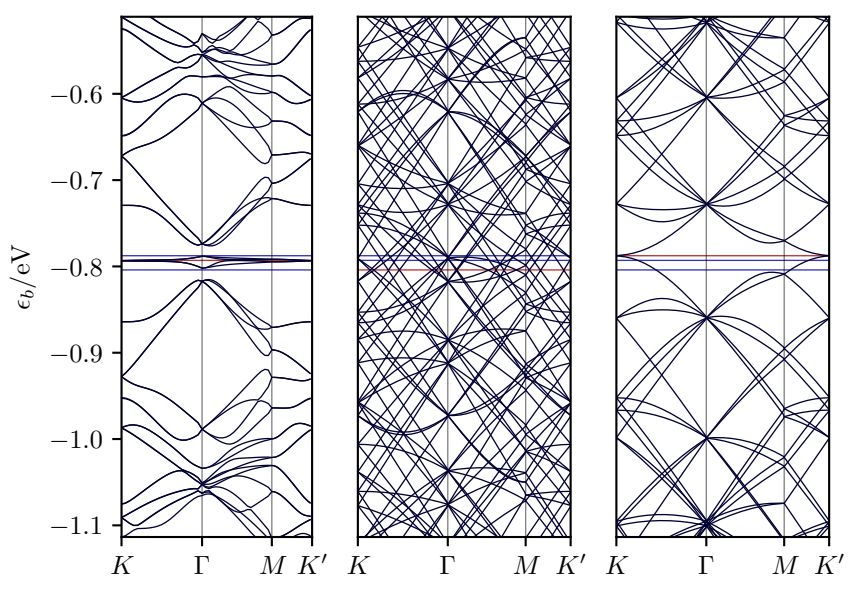

FIG. 1. Band structure in the moire Brillouin zone of the $31 / 32$ super cell. In the left plot we show the bands for the twisted bilayer with $\theta=1.05^{\circ}$, in the middle for the AAstacked and in the right plot for the AB-stacked 31/32-system. The red lines are the three Fermi energies at charge neutrality, the blue lines are the other systems' Fermi energies as a reference. The bandwidth of the four flat bands for the twisted bilayer is approximately $16 \mathrm{meV}$.

trons with opposite spins residing on carbon sites $i$,

$$
H_{U}=U \sum_{i} n_{i, \uparrow} n_{i, \downarrow}
$$

This interaction choice represents of course a major simplification with respect to the true long-range Coulomb interactions that are present in the experimental system. Actually, there are good cRPA estimates for the non-local Wannier-state interaction for mono-and bilayer graphene, also taking into account the embedding in an insulating substrate such as $\mathrm{hBN}^{30}$. However, it is also known that the primary instability tendencies are not affected qualitatively by the non-local terms. The latter terms only reduce the strength of the instability and could actually be absorbed into a redefined effective onsite repulsion $U^{* 229}$. Here we take the point of view that we just compare the AF instability trends for various bilayer configurations and by this avoid to pin down the ordering strength quantitatively on an absolute scale. Furthermore, recent $\mathrm{QMC}^{52}$ and $\mathrm{fRG} \mathrm{53}^{53}$ studies have given evidence that the leading instabilities are not changed by the non-local parts of the interaction and that the dominant instability for sufficient low-energy density of states is of AF-SDW type. This in turn can be well modeled with the Hubbard- $U$-only interaction. From our comparison of non-twisted and twisted systems we learn about how and how much the instability tendencies differ in these systems. 


\section{EFFECTIVE RPA THEORY FOR THE MAGNETIC ORDERING}

The magnetic ordering in a large number of correlated electron systems can be analyzed in simple terms by using the random-phase approximation (RPA) for the magnetic susceptibility $\hat{\chi}(\boldsymbol{q}, \nu)$. In this textbook-style approach, the transverse (up-down) spin channel, the spin susceptibility in the moiré unit cell can be written as

$$
\hat{\chi}^{z z}(q)=\hat{\chi}_{0}(q)\left[\mathbb{1}+U \hat{\chi}_{0}(q)\right]^{-1},
$$

where the matrices with the hat-symbol have $N_{M}$ components, running over all $N_{M}$ sites in the moiré unit cell. $\hat{\chi}_{0}(q)$ is the bare particle-hole bubble, with the matrix elements

$$
\begin{aligned}
\hat{\chi}_{0, i j}(\boldsymbol{q}, \nu)= & \frac{1}{N} \sum_{\boldsymbol{k}, b, b^{\prime}} \frac{n_{F}\left(\epsilon_{b}(\boldsymbol{k}+\boldsymbol{q})\right)-n_{F}\left(\epsilon_{b^{\prime}}(\boldsymbol{k})\right)}{-i \nu+\epsilon_{b}(\boldsymbol{k}+\boldsymbol{q})-\epsilon_{b^{\prime}}(\boldsymbol{k})} \\
& \cdot u_{i b}(\boldsymbol{k}+\boldsymbol{q}) u_{j b}^{*}(\boldsymbol{k}+\boldsymbol{q}) u_{i b^{\prime}}^{*}(\boldsymbol{k}) u_{j b^{\prime}}(\boldsymbol{k})
\end{aligned}
$$

According to this formula, the calculation of the $N_{M}^{2}$ matrix elements $\hat{\chi}_{0, i j}$ using the two internal summations $b, b^{\prime}$ over $N_{M}$ bands each would cause an effort $\mathcal{O}\left(N_{M}^{4}\right)$. However, by computing band-summed nonlocal Green's functions $G_{i j}\left(i \omega_{n}\right)$ and doing the Matsubara sums numerically afterwards for products $G_{i j}\left(i \omega_{n}\right) G_{j i}\left(i \omega_{n}\right)$, one can compute $\hat{\chi}_{0, i j}(\boldsymbol{q}, \nu=0)$ with effort $\mathcal{O}\left(N_{M}^{3} N_{\omega}\right)$ with $N_{\omega} \sim 500$ positive Matsubara frequencies on an appropriately chosen grid.

A divergence of the susceptibility (2) is obtained when an eigenvalue of $\hat{\chi}_{0}(\boldsymbol{q}, \nu)$ becomes $-1 / U$, e.g. when the temperature $T$ is lowered or the interaction strength $U$ is increased. This criterion is the generalization of the wellknown Stoner criterion for magnetic ordering. Note that, in order to keep the numerical effort limited, we compute the bubble diagram using 8 or 18 points in the irreducible moiré Brillouin zone. We have checked that the contributions of the lowest Matsubara frequencies do not change significantly if we use up to 72 points. Furthermore, we ignore possible selfenergy effects on the internal lines of the RPA bubbles.

Alternatively, we can Hubbard-Stratonovitch-decouple the Hubbard interaction in the functional integral formalism using a site-dependent magnetization $m_{i}(\tau)$. Then the fermions can be integrated out. The quadratic term in the magnetization then reads (generalizing, e.g., Ref. 54, and using $q=(\boldsymbol{q}, i \nu))$

$$
S^{(2)}=\frac{U}{4} \sum_{\substack{i j \\ q}} m_{i}^{*}(q)\left[\mathbb{1}+U \chi_{i j}(q)\right] m_{j}(q)
$$

Again, at sufficiently low temperatures $T$ or for large enough interaction strength $U$ and for $\nu=0$, the hermitian matrix in the square brackets will no longer be positive definite and at least one eigenvalue $\lambda_{0}$ with eigenvector $m_{i}^{(0)}$ will become smaller than zero. Then one can expect a nonzero magnetization $m_{i} \propto m_{i}^{(0)}$ to develop spontaneously. This onset of magnetic ordering is equivalent to the above-mentioned criterion for the divergence of the interacting susceptibility. It first happens at $\nu=0$, as the particle-hole bubble is largest then. In the fermionic action, $m_{i}$ couples linearly to the spin- $z$ component of the electrons. This can cause a gap in the spectrum around the Fermi level, depending on the dispersion and band filling. Previous studies ${ }^{37 / 55}$ of monoand nontwisted bilayer graphene have shown that the preferred ordering is at $\boldsymbol{q}=0$, with a staggered eigenvector with a sign change between the nearest neighbors on the honeycomb lattice and between the layers in the case of the AB-stacked bilayer. The relative size of the ordered moments in meanfield and QMC-studies $\sqrt{36}$ agree qualitatively with that found in the leading eigenvector in $\hat{\chi}$ in the $\mathrm{fRG}^{37}$, i.e. it is also larger on those sites that are not connected by the interlayer hopping. This is to be expected, as the weight of the bands touching at the Fermi level is higher on these sites than on the sites coupled by the interlayer hopping.

\section{CRITICAL INTERACTION STRENGTH FOR INSTABILITY}

In a first step we reanalyze the instability tendencies of the non-twisted bilayers with $\mathrm{AB}$ and AA stacking, by tracking for which parameters we find the divergence of the interacting susceptibility 2 described in the last section. For the 'plain-vanilla' version with nearest-neighbor hopping only and without remote hoppings, the SDW instability was studied in detail e.g. in Ref. 36, using RPA and QMC. Here we add to this by including the remote hopping processes as described by the tight-binding modeling of Ref. 11. These additional hoppings remove quadratic band crossing points in the case of the $\mathrm{AB}$ stacking and also alter the dispersion in the AA case. Hence, we no longer find instabilities at infinitely small $U$ as in some previous studies. The critical onsite- $U$ s are shown for a range of temperatures in Fig. 2.

As mentioned above, the possibility of a antiferromagnetic ordering on the $\mathrm{C}-\mathrm{C}$ bond scale for the twisted bilayer was already shown in selfconsistent studies for onsite interactions in Ref. 50. Here we ask if this instability is stronger in the twisted system compared to the $\mathrm{AB}$ or AA bilayer. In the same Fig. 2 we hence add the $U_{c^{-}}$ values for the $\theta=1.05^{\circ}$ twisted system. For the chargeneutral system we will show below that the instability is also essentially of antiferromagnetic type. We observe that for higher $T$ the $U_{c}$-values lie between those for AA and $\mathrm{AB}$ stacking, while they deviate significantly to lower $U$ at smaller $T$ s for the charge-neutral twisted bilayer. This shows that the twisted system at charge neutrality is even more susceptible to a magnetic instability than the non-twisted systems. The deviation occurs on temperatures scales of $1 \mathrm{meV}$, or $10 \mathrm{~K}$, which is the temperature range when the flat bands are resolved. Hence this 


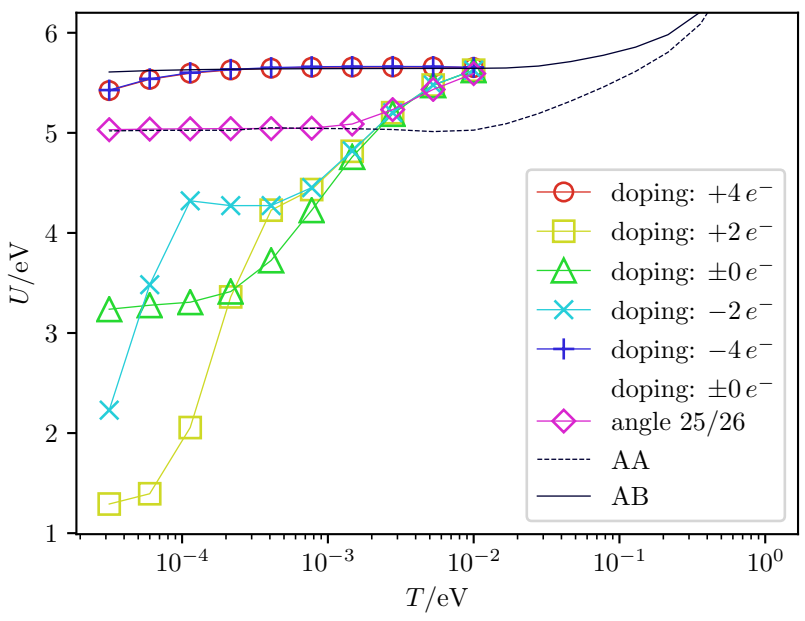

FIG. 2. Critical RPA onsite interaction strength vs. temperature for the $\boldsymbol{q}=0$ instability in the AA (dashed) and $\mathrm{AB}$ (solid line) bilayer without twist, and for the 1.05-degree twisted system and different fillings of the flat bands around the Fermi level, including remote hoppings as given in Ref. 11. In addition, the $25 / 26$ twisted system $\left(\theta=1.30^{\circ}\right)$ at charge neutrality is shown.

finding is not unexpected. Consistently, somewhat away from the magic angle, at $\theta=1.30^{\circ}$, the enhancement is less marked.

Interestingly, as shown in Fig. 2, the $U_{c}$ values for the \pm 2 -doped systems are even lower than the ones for the charge-neutral system. We see below that the ordering pattern is also different for those cases, but we cannot offer a simple intuitive explanation for this here. In any case it supports the statement that the flat bands of twisted bilayer graphene exhibit a rather rich and versatile physics.

\section{SPATIAL STRUCTURE OF THE MAGNETIC ORDERING}

Next we discuss the type of magnetic order that is suggested by this RPA analysis. This becomes clear from the spatial structure of the eigenvector that belongs to the leading eigenvalue of the instability.

First, let us consider the charge-neutral system. In Fig. 3 we plot the absolute value of the eigenvector in the moire unit cell for two temperatures. We see that the eigenvector is larger in the AA regions in the corner of the rhomb-shaped unit cell. At the higher $T$ the eigenvector is somewhat more extended away from the AA regions compared to the data at lower $T$, in particular along the diagonal which separates the $\mathrm{AB}$ region from the $\mathrm{BA}$ region. In the plots below, in Fig. 4, we show lines cuts of the eigenvector from the AA region through $\mathrm{AB}$ and $\mathrm{BA}$ regions to the other $\mathrm{AA}$ region on the other side of the unit cell in one layer. We clearly observe the sign oscillations, which identify the instability as to- ward antiferromagnetic (AF) order with opposing signs of the order parameter on A and B sublattice in the same layer. This order also occurs in homogeneous fashion for the nontwisted $\mathrm{AA}$ and $\mathrm{AB}$ bilayers. It appears that the twisted system just inherits this order parameter from the non-twisted bilayers, with additional modulation owing to the inhomogeneous distribution of the density of states at lowest energies.

The more precise comparison between the two eigenvectors at different temperatures reveals that at the lower $T \lesssim 2 \mathrm{meV}$, which is of the order of the van Hove $M$-point energy in the flat-band dispersions, the staggered magnetic order changes its registry along the path from the $\mathrm{AA}$ to the $\mathrm{AB}$ or $\mathrm{BA}$ region. This is clearly visible as a node in the absolute value of the order parameter. Besides the overall modulation through the unit cell, this is another alteration of the ordering in the twisted systems, indicating that the flat bands induce additional physical complexity.

In order to understand the impact of the magnetic order on the electronic spectrum, we insert the leading eigenvector of the susceptibility as our guess for the meanfield that occurs in the diagonal of the hopping Hamiltonian of the electrons. At charge neutrality, we easily find a gap opening at the Fermi level due to the ordering. Two cases at different temperatures, one for the nodeless AF ordered state and one for the nodal state a lower $T$ are shown in Fig. 5 We expect that a self-consistent solution of the magnetic meanfield theory would give similar spectra. On the quantitative side, our gaps come out an order of magnitude larger than the activation energies observed experimentally $\underline{4}$. Note however that we just inserted $U / 4$ times the eigenvector into the Hamiltonian as a naïve guess. No attempt to obtain a selfconsistent solution was made. Furthermore, various fluctuations and renormalizations may influence the actual gap size. Qualitatively, our data supports the expectation that the AF ordered states should be gapped and hence exhibit insulator-like transport physics. Next we move to the doped system with $\pm n$ and $|n| \leq 4$ electrons per moiré unit cell. Here the leading eigenvector at low $T$ and doping +2 is shown in Fig. 6 .

We observe that while there is still some staggering on the eigenvector along the trace through the unit cell, the overall sign of the eigenvector is the same in most of the unit cell except for islands with oscillating sign near the $\mathrm{AB}$ and $\mathrm{BA}$ regions. This means that the leading instability is of ferromagnetic (FM) type now, again with strongest weight in the AA regions. Again, we can insert the eigenvector as order parameter into the hopping Hamiltonian. It turns out that the spectra at \pm 2 become gapped rather readily but that it is hard to induce a gap for odd-integer dopings via this simple route. Here we would have to invoke a stronger coupling or additional mechanisms beyond our present scheme.

Notably, at somewhat higher temperatures, we still find the antiferromagnetic solution, and only at lower $T$ the FM eigenvalue becomes strongest. Again this sup- 

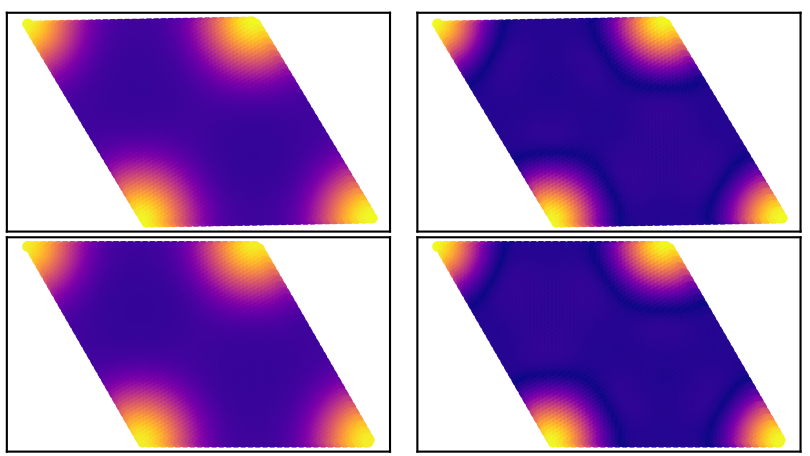

FIG. 3. RPA data at charge neutrality: absolute value of the leading eigenvector proportional to the magnetic order parameter in the two-layer rhomb-shaped unit cell (blue to yellow: smallest to largest absolute value, upper plots: upper (rotated) layer, lower plots: lower layer). The AA-regions are in the corners of the rhomb, the AB- and BA-region on the diagonal from the upper left to the lower right corner at one and two thirds distance. Left plot: intermediate temperature $T=10 \mathrm{meV}$, right plot: low temperature $T=0.408 \mathrm{meV}$.

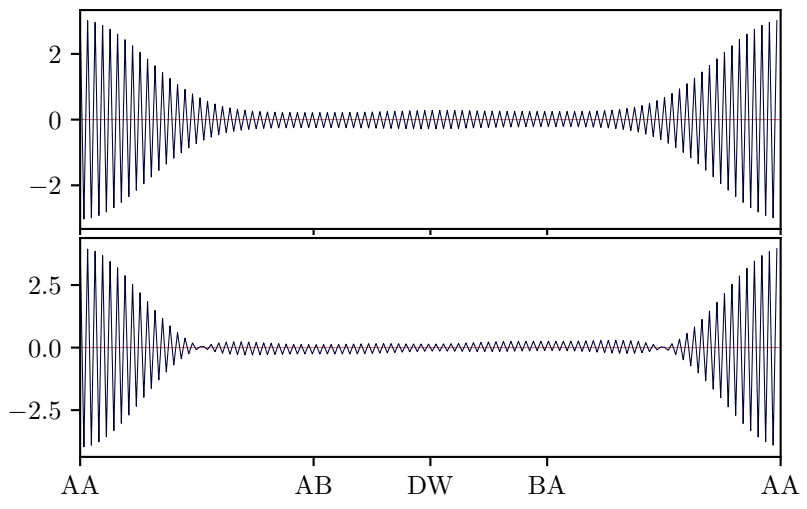

FIG. 4. RPA data at charge neutrality: line cut of the leading eigenvector proportional to the magnetic order parameter through the rhomb-shaped unit cell in one layer, starting in the AA-regions in the corners of the rhomb through the ABand BA-region on the diagonal to the next AA-region. Upper plot: intermediate temperature $T=10 \mathrm{meV}$, lower plot: low temperature $T=0.408 \mathrm{meV}$ with an additional node of the order parameter near the AA regions.

ports the idea that the flat-band physics is quite rich. The higher- $T$ state is the inherited order from the nontwisted system, which is dominated by the bulk of the spectrum, while at lower $T$ the flat bands add additional features and alter the ordering pattern to ferromagnetic alignment.

\section{TENTATIVE PHASE DIAGRAMS}

We can summarize our findings in a tentative RPA phase diagram for the magnetic ordering ordering in the magic-angle bilayer system. This is shown for selected
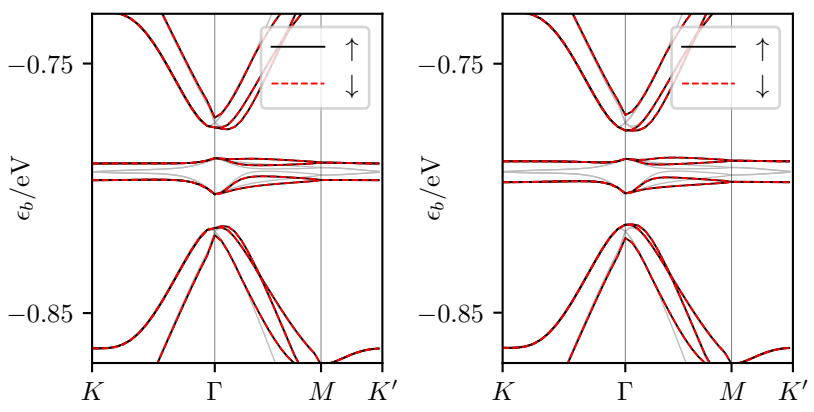

FIG. 5. Magnetic band structure obtained by inserting the site- and spin-dependent leading eigenvector multiplied by $U / 4$ (with $U \approx 2 \mathrm{eV}$ ) into the diagonal of the hopping Hamiltonian. Left plot: intermediate temperature $T=10 \mathrm{meV}$, gap size $\Delta \approx 6.70 \mathrm{meV}$, right plot: low temperature $T=$ $0.03 \mathrm{meV}$, gap size $\Delta \approx 8.36 \mathrm{meV}$. The path goes through the reduced Brillouin zone of the moiré lattice. The nonmagnetic bands are indicated by the faint lines.
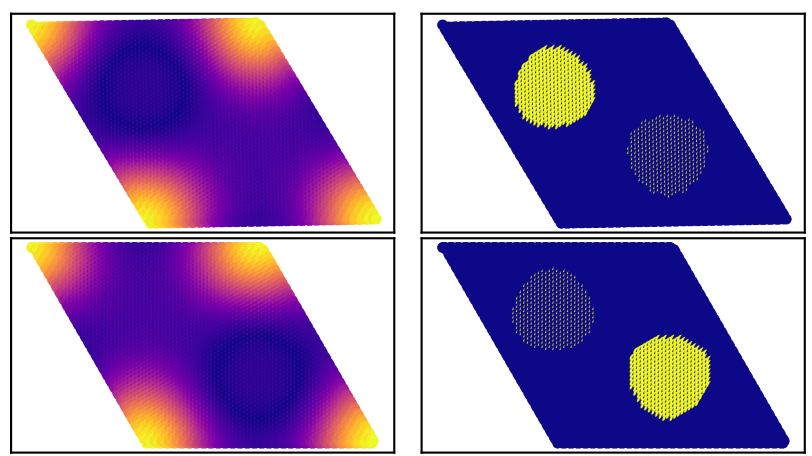

FIG. 6. Absolute value (left) and sign (right) of the leading eigenvector for the +2 doped system and temperature $T=$ $0.215 \mathrm{meV}$.

even integer dopings in Fig. 8 for a larger number of temperatures. We also include dopings other than \pm 2 in Fig. 9, where we scan the critical interaction strength as a function of the chemical potential $\mu$ for two low temperatures. According to this data, there is an enhanced ordering tendency for the filling range between -3 to +2 electrons around charge neutrality at filling 0 . Now, if the relevant onsite interaction strength $U$ would be around 4 or $5 \mathrm{eV}$, as argued in Ref. 30 , this would re-

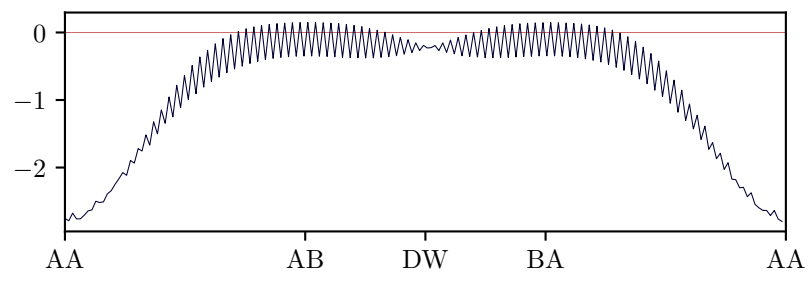

FIG. 7. Line cut of the leading eigenvector for the +2 doped system. The path is again diagonal through the rhombshaped unit cell from the top left to the bottom right corner. 


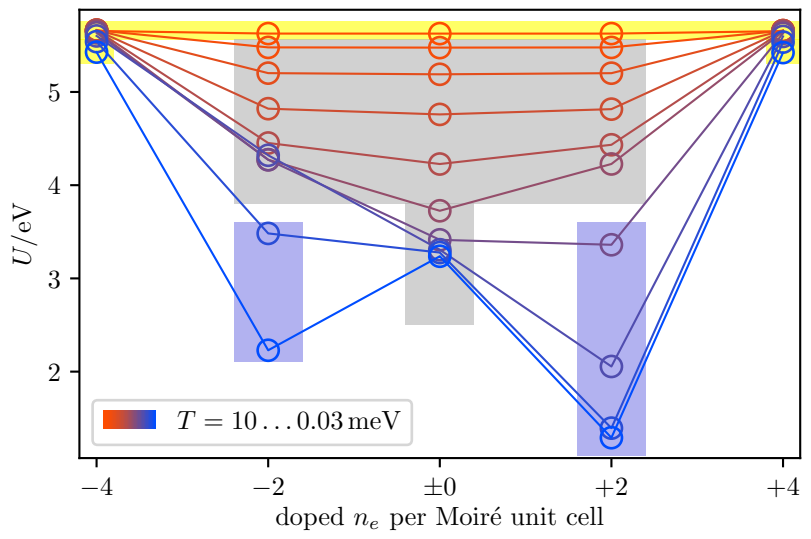

FIG. 8. Tentative magnetic RPA phase diagram for the filling range of even integer doping of up to \pm 4 electrons per moiré unit cell. The lines show the threshold interaction strengths for magnetic ordering for different temperatures. Lower values correspond to increased instability toward ordering. The color shading indicates the type of magnetic order - yellow: nodeless antiferromagnetic order, grey: antiferromagnetism with real-space node in order parameter, blue: ferromagnetic order.

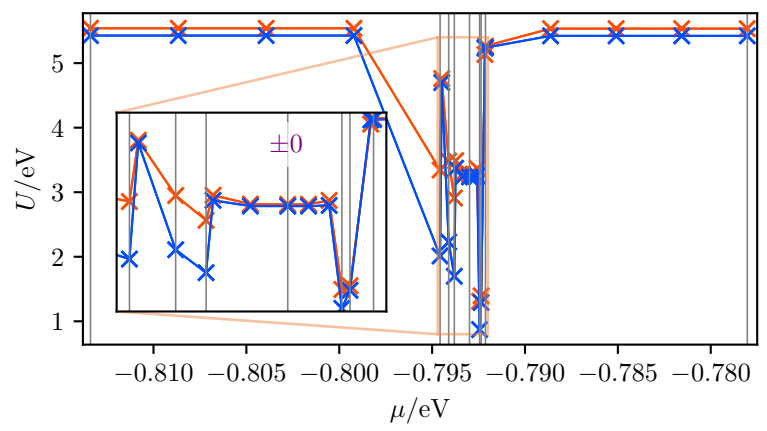

FIG. 9. Critical onsite interaction strength $U$ versus chemical potential $\mu$, indicating the instability tendency for dopings in the filling range \pm 4 electrons per moiré unit cell. The vertical lines indicate integer fillings $-4,-3, \ldots, 4$ with charge neutrality (filling 0 ) at $\mu \approx 0.793 \mathrm{eV}$. Red line: temperature $T=0.06 \mathrm{meV}$, blue line: temperature $T=0.03 \mathrm{meV}$. The inset shows the same data on an enlarged $\mu$-scale. All integer dopings -3 to +2 electrons per moiré unit cell show an increased magnetic ordering tendency.

sult in a window for magnetic order between fillings -3 and +2 electrons per moiré unit cell. Then, the fillings 0 and \pm 2 should be insulating, as then the Fermi level would lie between the respective bands. For other fillings, including \pm 1 and +3 electrons per moiré unit cell, one should have a metallic state, potentially with a magnetically ordered background. Of course, additional effects such as increased coupling to the order parameter or magnetic disorder could render these states insulating as well. Outside the window -3 to +2 electrons, we do not find amplified ordering tendencies. In particular, within this simple picture, we cannot explain the potential insulating state at +3 electrons, because the interaction tendency is not strongly enhanced there. The insulator at \pm 4 is believed to be a trivial band insulator, which remains unchanged by our analysis.

\section{DISCUSSION AND CONCLUSIONS}

In this random-phase approximation (RPA) study of the full $\pi$-band spectrum, we have compared the instability tendency of the twisted graphene bilayer to the untwisted AB- and AA-stacked version. The main goal was to determine the ground state order, and from that we derived estimates of the low-energy properties, with the goal to understand the nature of the insulating states in magic-angle twisted bilayer graphene.

We found that at intermediate temperatures above $\sim 20 \mathrm{~K}$ the instability strength of magic-angle twisted bilayers at charge neutrality toward antiferromagnetic (AF) ordering lies close to that of the non-twisted AB-stacked or AA-stacked systems. For lower temperatures, the flat bands of the twisted systems strongly enhance the RPA instability tendency, and the corresponding threshold interaction strengths become quite small and fall below the theoretical estimates for the effective onsite interaction in standard graphene systems $28-30$. Thus it is very likely that the twisted systems may indeed order in this way at low $T$, at least locally. We can understand this AF instability inherited from the nontwisted $\mathrm{AB}$ bilayers. Note that various experiments showing low-temperature gap openings in freely suspended Bernal( $\mathrm{AB})$-stacked graphene were interpreted in terms of $\mathrm{AF}$ ordering 3143 . Of course, the embedding in $\mathrm{hBN}$ of the twisted system may cause some additional screening, but in general the change in the short-range interaction parameters due to the $\mathrm{hBN}$ will be limited 30 . Hence it is not implausible to consider the possibility that the twisted bilayers order in an analogous way even if they are encapsulated in hBN. It would be very interesting to search for the staggered and unit-cell modulated spin correlations, potentially with spin-resolved scanning tunneling techniques. Away from charge neutrality, the main magnetic ordering tendency turned out to be ferromagnetic (FM) when the temperature was lower than the typical van-Hove-singularity flat-band dispersion energies. This points to the impact of the flat bands in inducing FM correlations instead of the AF order. Of course, it would be interesting to understand the relation of this effect to known cases of flat-band ferromagnetism 56 .57.

Discussing potential caveats of our approach, one should mention that the RPA used here clearly runs the danger of overestimating the ordering tendencies. Channel coupling and selfenergy corrections, as well as nonlocal interactions will reduce the energy scales for ordering and increase the threshold interaction strength needed for the instabilities. We are currently working to implement a functional renormalization group study of these systems, in order to asses these issues more thor- 
oughly. First results support the RPA picture. Moreover, the differences between RPA and more controlled approaches in this context are known to be limited from studies of nontwisted systems ${ }^{36}$. On the positive side, compared to many studies focusing on just four lowenergy bands, our study is far more quantitative as it does not ignore a large portion of the degrees of freedom. If the flat low-energy bands show a different physics from that of the nontwisted layers, it should be visible. Indeed, we found that small doping with just one or two electrons per unit cell leads to a qualitatively different, ferromagnetically ordered ground state.

We furthermore argued that a (partial) sequence of insulating states can be understood by inserting the corresponding suggested orders as meanfields, at least for the charge-neutral and the \pm 2 -doped cases. There, if the order parameter is large enough, the flat bands split up such as to give gaps for integer band fillings. If the density is away from these even integer fillings, the splitup bands of the magnetically ordered state are partially filled, and the system remains metallic. This opens windows for superconductivity in between the integer fillings. Note that our current approach is not suitable to search for superconductivity, but at least it allows one to understand how metallic and insulating situations can arise as a function of he electron density in the flat bands. The picture given here would mean that the superconductivity arises away from integer fillings in addition to or in the background of the magnetic ordering suggested by the RPA. The mechanism would then either be phononic or electronic, by the fluctuations of the magnetic order. As the magnetic order appears to be doping-dependent, there is the actual possibility of distinct superconducting states for different dopings. As the magnetic order breaks time-reversal and spin rotational symmetry, the superconducting states could be unconventional, even if they are mediated by phonons. Exploring these possibilities will be the content of future work.

We also commented on the fact that our current study cannot readily explain insulating states a odd integer band fillings \pm 1 and +3 . For these, one may have to include additional symmetry breakings of self-energy effects. In addition to that, charge redistributions $\sqrt{58}$ and order at nonzero wavevectors varying on the moiré scal $\mathrm{e}^{\sqrt{50}}$ can lead to additional physics not accounted for here. It will be interesting to study how these effects add to the Angström-scale magnetic order found here.

We thank L. Classen, A. Honecker, J. Lado, A. MacDonald, M. Morgenstern, and M. Scherer for discussions. The German Science Foundation (DFG) is acknowledged for support through RTG 1995, and JARA-HPC for granting computing time.
1 Y. Cao, V. Fatemi, A. Demir, S. Fang, S. L. Tomarken, J. Y. Luo, J. D. Sanchez-Yamagishi, K. Watanabe, T. Taniguchi, E. Kaxiras, et al., Nature 556, 80 (2018).

2 Y. Cao, V. Fatemi, S. Fang, K. Watanabe, T. Taniguchi, E. Kaxiras, and P. Jarillo-Herrero, Nature 556, 43 (2018).

3 M. Yankowitz, S. Chen, H. Polshyn, K. Watanabe, T. Taniguchi, D. Graf, A. F. Young, and C. R. Dean, arXiv preprint arXiv:1808.07865 (2018).

${ }^{4}$ X. Lu, P. Stepanov, W. Yang, M. Xie, M. A. Aamir, I. Das, C. Urgell, K. Watanabe, T. Taniguchi, and G. Zhang, arXiv e-prints , arXiv:1903.06513 (2019), arXiv:1903.06513 [cond-mat.str-el]

G. Trambly de Laissardière, D. Mayou, and L. Magaud, Nano Letters 10, 804 (2010), pMID: 20121163, https://doi.org/10.1021/nl902948m.

6 R. Bistritzer and A. H. MacDonald, Proceedings of the National Academy of Sciences 108, 12233 (2011),

https://www.pnas.org/content/108/30/12233.full.pdf

7 J. M. B. Lopes dos Santos, N. M. R. Peres, and A. H. Castro Neto, Phys. Rev. B 86, 155449 (2012)

8 G. Li, A. Luican, J. L. Dos Santos, A. C. Neto, A. Reina, J. Kong, and E. Andrei, Nature Physics 6, 109 (2010).

9 I. Brihuega, P. Mallet, H. González-Herrero, G. Trambly de Laissardière, M. M. Ugeda, L. Magaud, J. M. GómezRodríguez, F. Ynduráin, and J.-Y. Veuillen, Phys. Rev. Lett. 109, 196802 (2012)

${ }^{10}$ Y. Kim, P. Herlinger, P. Moon, M. Koshino, T. Taniguchi, K. Watanabe, and J. H. Smet, Nano Letters 16, 5053 (2016), pMID: 27387484, https://doi.org/10.1021/acs.nanolett.6b01906

11 M. Koshino, N. F. Q. Yuan, T. Koretsune, M. Ochi,
K. Kuroki, and L. Fu, Phys. Rev. X 8, 031087 (2018)

12 J. Kang and O. Vafek, Phys. Rev. X 8, 031088 (2018)

13 H. C. Po, L. Zou, A. Vishwanath, and T. Senthil, Phys. Rev. X 8, 031089 (2018).

${ }^{14}$ J. F. Dodaro, S. A. Kivelson, Y. Schattner, X. Q. Sun, and C. Wang, Phys. Rev. B 98, 075154 (2018)

15 B. Roy and V. Juričić, Phys. Rev. B 99, 121407 (2019)

${ }^{16}$ H. Guo, X. Zhu, S. Feng, and R. T. Scalettar, Phys. Rev. B 97, $235453(2018)$

If Q.-K. Tang, L. Yang, D. Wang, F.-C. Zhang, and Q.-H. Wang, Phys. Rev. B 99, 094521 (2019)

18 C.-C. Liu, L.-D. Zhang, W.-Q. Chen, and F. Yang, Phys. Rev. Lett. 121, 217001 (2018).

${ }^{19}$ H. Isobe, N. F. Q. Yuan, and L. Fu, Phys. Rev. X 8, 041041 (2018)

20 J. W. F. Venderbos and R. M. Fernandes, Phys. Rev. B 98, 245103 (2018)

${ }^{21}$ Y. Da Liao, Z. Y. Meng, and X. Y. Xu, arXiv preprint arXiv:1901.11424 (2019).

22 D. M. Kennes, J. Lischner, and C. Karrasch, Phys. Rev. B 98, 241407 (2018)

${ }^{23}$ Y.-P. Lin and R. M. Nandkishore, arXiv preprint arXiv:1901.00500 (2019).

24 L. Classen, C. Honerkamp, and M. M. Scherer, Phys. Rev. B 99, 195120 (2019)

25 J. M. Pizarro, M. Rösner, R. Thomale, R. Valentí, and T. O. Wehling, arXiv e-prints , arXiv:1904.11765 (2019), arXiv:1904.11765 [cond-mat.str-el]

26 D. Baeriswyl and K. Maki, Phys. Rev. B 31, 6633 (1985)

27 R. J. Bursill, C. Castleton, and W. Barford, Chemical Physics Letters 294, 305 (1998) 
${ }^{28}$ T. O. Wehling, E. Şaşıŏlu, C. Friedrich, A. I. Lichtenstein, M. I. Katsnelson, and S. Blügel, Phys. Rev. Lett. 106, 236805 (2011)

29 M. Schüler, M. Rösner, T. O. Wehling, A. I. Lichtenstein, and M. I. Katsnelson, Phys. Rev. Lett. 111, 036601 (2013)

30 M. Rösner, E. Şaşığlu, C. Friedrich, S. Blügel, and T. O. Wehling, Phys. Rev. B 92, 085102 (2015).

31 J. Velasco Jr, L. Jing, W. Bao, Y. Lee, P. Kratz, V. Aji, M. Bockrath, C. N. Lau, C. Varma, R. Stillwell, D. Smirnov, F. Zhang, J. Jung, and A. H. MacDonald, Nature Nanotechnology 7, 156 EP (2012)

32 W. Bao, L. Jing, J. Velasco Jr, Y. Lee, G. Liu, D. Tran, B. Standley, M. Aykol, S. B. Cronin, D. Smirnov, M. Koshino, E. McCann, M. Bockrath, and C. N. Lau, Nature Physics 7, 948 EP (2011).

33 F. Freitag, J. Trbovic, M. Weiss, and C. Schönenberger, Phys. Rev. Lett. 108, 076602 (2012), arXiv:1104.3816 [cond-mat.mes-hall]

${ }_{34}$ A. Veligura, H. J. van Elferen, N. Tombros, J. C. Maan, U. Zeitler, and B. J. van Wees, Phys. Rev. B 85, 155412 (2012)

35 J. Nilsson, A. H. Castro Neto, N. M. R. Peres, and F. Guinea, Phys. Rev. B 73, 214418 (2006).

36 T. C. Lang, Z. Y. Meng, M. M. Scherer, S. Uebelacker, F. F. Assaad, A. Muramatsu, C. Honerkamp, and S. Wessel, Phys. Rev. Lett. 109, 126402 (2012)

37 M. M. Scherer, S. Uebelacker, and C. Honerkamp, Phys. Rev. B 85, 235408 (2012)

38 M. M. Scherer, S. Uebelacker, D. D. Scherer, and C. Honerkamp, Phys. Rev. B 86, 155415 (2012)

39 F. Zhang, J. Jung, G. A. Fiete, Q. Niu, and A. H. MacDonald, Phys. Rev. Lett. 106, 156801 (2011)

${ }^{40}$ R. E. Throckmorton and O. Vafek, Phys. Rev. B 86, 115447 (2012)

${ }^{41}$ M. Kharitonov, Phys. Rev. B 86, 195435 (2012).

42 A. L. Grushina, D.-K. Ki, M. Koshino, A. A. L. Nicolet, C. Faugeras, E. McCann, M. Potemski, and A. F. Mor- purgo, Nature Communications 6, 6419 EP (2015)

43 Y. Nam, D.-K. Ki, D. Soler-Delgado, and A. F. Morpurgo, Science 362, 324 (2018), arXiv:1811.10212 [cond-mat.meshall].

${ }^{44}$ K. Myhro, S. Che, Y. Shi, Y. Lee, K. Thilahar, K. Bleich, D. Smirnov, and C. N. Lau, 2D Materials 5, 045013 (2018)

45 J. Martin, B. E. Feldman, R. T. Weitz, M. T. Allen, and A. Yacoby, Phys. Rev. Lett. 105, 256806 (2010).

46 R. T. Weitz, M. T. Allen, B. E. Feldman, J. Martin, and A. Yacoby, Science 330, 812 (2010), https://science.sciencemag.org/content/330/6005/812.full.pdf.

47 A. S. Mayorov, D. C. Elias, M. Mucha-Kruczynski, R. V. Gorbachev, T. Tudorovskiy, A. Zhukov, S. V. Morozov, A. K. Katsnelson, M. I. Geim, and K. S. Novoselov, Science 333, 860 (2011), https://science.sciencemag.org/content/333/6044/860.full.pdf.

48 Y. Lemonik, I. Aleiner, and V. Falko, Physical Review B: Condensed Matter and Materials Physics 85, (2012)

49 L. Classen, M. M. Scherer, and C. Honerkamp, Phys. Rev. B 90, 035122 (2014)

5o L. A. Gonzalez-Arraga, J. L. Lado, F. Guinea, and P. SanJose, Phys. Rev. Lett. 119, 107201 (2017)

51 P. Moon and M. Koshino, Phys. Rev. B 85, 195458 (2012)

52 H.-K. Tang, E. Laksono, J. N. B. Rodrigues, P. Sengupta, F. F. Assaad, and S. Adam, Phys. Rev. Lett. 115, 186602 (2015)

53 D. S. de la Peña, J. Lichtenstein, C. Honerkamp, and M. M. Scherer, Phys. Rev. B 96, 205155 (2017)

54 J. A. Hertz, Phys. Rev. B 14, 1165 (1976).

55 C. Honerkamp, Phys. Rev. Lett. 100, 146404 (2008)

56 A. Mielke and H. Tasaki, Communications in Mathematical Physics 158, 341 (1993).

57 R. Hlubina, S. Sorella, and F. Guinea, Phys. Rev. Lett. 78, 1343 (1997)

58 F. Guinea and N. R. Walet, Proceedings of the National Academy of Sciences 115, 13174 (2018), https://www.pnas.org/content/115/52/13174.full.pdf 